\title{
MODELLING OF QUEUING PROCESS AT AIRPORT CHECK-IN SYSTEM: A CASE STUDY OF MANCHESTER AND LEEDS-BRADFORD AIRPORTS
}

\author{
P. T. Adeke* \\ Department of Civil EngineEring, University of Agriculture MaKURdi, MaKURdi, BENUE State, NiGERIA. \\ E-mail address: adeke.pt@outlook.com
}

\begin{abstract}
This study built a Simulation Model (SM) using SimEvents toolbox in MATLAB for implementing Analytical Models (AM) of queuing process at airport check-in system. Air travel demand data for Manchester and Leeds-Bradford airports in 2014 were adopted for validation of the model. There was no statistical difference between utilisation factor (UF) and service times of AM and SM outputs. Differences in AM and SM outputs for average queue length, average waiting time on queue and average number of arrivals and throughputs were attributed to variations in discrete time events considered by SM in contrary to the AM which assumed constant values for the process. The SM exhibited stochastic behaviour which actually depicts reality hence produces more reliable results. Stochastic analysis methods are therefore recommended for queuing analysis to achieve accurate results. The SM is therefore recommended to give Airport managers prior knowledge of system performance for planning and improved level of service (LOS) at airports.
\end{abstract}

Key words: Airport check-in system, discrete time events, analytical models, simulation model, SimEvents toolbox.

\section{INTRODUCTION}

The concept of queuing is generally employed by waiting customers in service delivery systems to ensure orderliness and efficient operations of the system [1-3]. The concept was first used for the analysis of telephone call traffic in 1913 [4-6].

Following the annual growth in aviation travel demand in the world [7 - 9], longer queues due to congested travellers waiting for check-in services at airports are anticipated $[10,11,9]$; this calls for efficient service management strategies that would ensure better Level of Service (LOS) for check-in process at airports [12 14]. LOS at the airport is the satisfaction derived from service facilities or operations by travellers [15].

Queues are characterised by available service units, service pattern which could be in series or parallel and queue discipline which could be; First-In-First-Out (FIFO) meaning who comes in first leaves earlier, LastIn-First-Out (LIFO) meaning, who comes in later leaves earlier. Random Service (RS) which means random selection of customers and priority rules where arrivals are being prioritised for services [3, 16 - 18].

The finite or infinite random arrival on queues is defined as stochastic process also known as Markov (or memoryless) process described by Poisson distribution. Service times are independently and identically distributed or exponentially distributed random variables; the process is expressed by Erlange model [17, 19 - 21].

Queuing models are described using probability distribution of inter-arrivals and service times, number of servers and queue discipline forming the Erlangian models $[3,22]$. Queuing models are categorised into deterministic, where inter-arrival times and service times are constant or Markovian where inter-arrival time and service times are exponentially distributed or independently and identically distributed (IID). Following Kendall's notation, the Poisson arrival process due to its exponential assumptions is expressed as $\mathrm{A} / \mathrm{B} / \mathrm{m} / \mathrm{K} / \mathrm{n} / \mathrm{D}$, where element $\mathrm{A}$ denotes the inter-arrival time distribution, $\mathrm{B}$ denotes service time distribution, $m$ denotes number of servers, $\mathrm{K}$ is the capacity of the system, $\mathrm{n}$ is the population and $\mathrm{D}$ is the service discipline employed in the system [18]. Element A, commonly presented as $\mathrm{M}$, is used to represent the Markov exponential distribution; other notations used include $G$ for general distribution and $D$ for deterministic distribution of inter-arrival or service times [20]. 
The principal aim of queue studies is to examine performance characteristics of service system using parameters such as; inter-arrival rate, service rate, number of servers, pattern of service, busy and idle times of servers, utilisation of servers, service discipline; other parameters of interest include; number of customers on queue and in system, waiting time on queue and time spent in system etc., used for determining the efficiency and capacity management strategy of the system $[1,2]$. The use of models for queue analysis aims at describing the process and to predict system's behaviour due to changes [22]. Based on its economic benefits, the performance of queuing system as influenced by system configuration and service protocol, are essential inputs to system managers for decision making $[9,23]$.

This study aims at modelling queuing process at airport check-in system to evaluate queuing performance variables using Analytical Models (AM) and implement the AM using Simulation Model (SM) in MATLAB, using SimEvent toolbox to ascertain the suitability of both models in queue studies.

The study adopted travel demand data for Manchester and Leeds-Bradford airports from the United Kingdom Civil Aviation Authority database.

\subsection{Analytical Models for Queuing Studies}

Previous researchers have examined queuing process extensively and developed analytical models used for examining queue performance $[3,4,16,20,24,25]$.

The random arrivals described by Poisson distribution are expressed such that, for number of arrivals $N(t)$ within time interval $[0, t]$ with arrival rate $\lambda$, the expression of time between successive arrivals is exponentially distributed with parameter $\mu$ and independent of previous arrivals. This is referred to as memoryless process whereby the probability that service completed at future time is independent of how long the customers stayed in the service facility [4].

The parameter for Poisson distribution of $N(t)$ is given as $\lambda t$, and the probability of each arrival in the system is given as Eq. (1);

$$
\begin{aligned}
P\{N(t)=k\}= & \frac{(\lambda t)^{k}}{k !} e^{-\lambda t} \quad \lambda>0 \text { and } k \\
& =0,1,2,3, \ldots
\end{aligned}
$$

The probability that there is no arrival within time interval is given as; $P(0)=e^{-\lambda t}$, such that the probability of having an arrival within time interval is given as Eq. (2);

$$
P(x)=1-e^{-\lambda t}
$$

The Poisson distribution is characterised by equal mean and equal variance; therefore, the probability of arrivals within time interval $\Delta t$ is expressed as Eq. (3);

$$
\begin{array}{r}
\operatorname{Pr}\{N(t,(t+\Delta t))\}=\lambda \Delta t-o(\Delta t) \\
\approx \lambda \Delta t \text { as }(\Delta t \rightarrow 0)
\end{array}
$$

According to [20] and [24], inter-arrival time of random entities $(X)$ described as stochastic process with nonnegative exponential distribution having parameter $\lambda>0$ and probability density function (pdf) is given as shown in Eq. (4);

$$
f(x)=\{X \leq x\}= \begin{cases}\lambda e^{-\lambda x} & x \geq 0 \\ 0 & x<0\end{cases}
$$

The cumulative distribution function (cdf) is given as shown in Eq. (5);

$$
F(x)=P\{X \leq x\}=\left\{\begin{array}{cc}
1-e^{-\lambda x} & x \geq 0 \\
0 & x<0
\end{array}\right.
$$

The mean value of $X$ and variance are expressed in Eqs. (6) and (7) respectively;

$$
\begin{aligned}
& E[X]=\int_{0}^{\infty} x f(x) d x=\frac{1}{\lambda} \\
& \operatorname{var}[X]=\int_{0}^{\infty} x^{2} f(x) d x-E(X)^{2}=\frac{1}{\lambda^{2}}
\end{aligned}
$$

Using integration by parts, the nth moment of $\mathrm{X}$ is given as Eq. (8);

$$
E\left[X^{n}\right]=\int_{0}^{\infty} x^{n} \lambda e^{-\lambda x} d x=\frac{n !}{\lambda^{n}}
$$

The forgetfulness property of the stochastic process is expressed using $\mathrm{T}$ the total waiting time and $\mathrm{t}$ the waited time given as Eq. (9);

$$
P[X \leq x+t \mid T>t]=\lambda e^{-\lambda(x-t)}
$$

According to [16], for each arrival in a service system, the next arrival occurs after an exponential time with parameter $\lambda+\mu$, and probability $\lambda /(\lambda+\mu)$. Therefore, the probability of completing service and the customer leaving is given as $\mu /(\lambda+\mu)$.

The performance of a single server in a multiple-server system with $m$ number of servers is such that, traffic intensity $\rho$ is expressed as the product of arrival intensity $\lambda$ and mean inter-arrival time $1 / m \mu$. Whenever $\rho>1$, the system is said to be overloaded, that is, random arrivals are relatively higher than departures, hence system is unsteady $[3,18]$.

For an $\mathrm{M} / \mathrm{M} / \mathrm{m}$ model, where $\mathrm{m}$ is the number of servers per queue, performance parameters are estimated using utilisation factor or demand intensity $\rho$ which is the percentage of time servers are busy during the process. For an M/M/1 model, $\rho$ is given as Eq. (10);

$$
\rho=\frac{\lambda}{\mu}
$$

For a multi-server system based on $\mathrm{M} / \mathrm{M} / \mathrm{m}$ model Eq. (10) is expressed as Eq. (11);

$$
\rho=\frac{\lambda}{m \mu}
$$

where, $m$ is the number of servers in the system. When $\lambda<\mu$ or $m \mu>\lambda$ such that $\rho<1$ for Eq. (10) and (11) respectively, the system is classified as a steady state or 
an equilibrium system; otherwise, unsteady or nonequilibrium where arrivals continue to increase indefinitely $[3,16]$. Demand intensity of the system at each arrival can be estimated using Eq. (12);

$$
\rho=\frac{N(t) \lambda}{m \mu}
$$

where, $N(t)$ equals the number of arrivals on queue at instantaneous time, $t$. For a single server model $M / M / 1$, [3] established that, total number of customers in the system within time limits $[0, t]$ is as derived in Eq. (13);

$$
\begin{gathered}
N(t)=A(0, t)-D(0, t)=\left[\frac{t}{1 / \lambda}\right]-\left[\frac{t-1 / \lambda}{1 / \mu}\right] \\
=[\lambda t]-\left[\mu t-\frac{\mu}{\lambda}\right]
\end{gathered}
$$

where A and D represent the number of arrivals and departures respectively. From Eq. (12) it is established that, total number of customers in the system based on $\mathrm{M} / \mathrm{M} / \mathrm{m}$ model could be estimated using Eq. (14);

$$
N(t)=A(0, t)-m D(0, t)=[\lambda t]-m\left[\mu t-\frac{\mu}{\lambda}\right]
$$

Using $M / M / m$ model, according to [16], steady state probability of a system is determined from a flow diagram where inflow and outflow sets of state $\{0,1, \ldots$ , $n-1\}$, and flow between two side-by-side states $n-1$ and $n$ is given as Eq. (15);

$$
\lambda p_{n-1}=\min (n, m) \mu p_{n}, n=1,2, \ldots
$$

By iteration, the probability of states $n$ and $n+m$ are given respectively as Eq. (16) and (17);

$$
\begin{aligned}
& \qquad p_{n}=\frac{(m \rho)^{n}}{n !} p_{0} \quad n=0,1, \ldots, m \\
& \text { and } \quad p_{m+n}=\rho^{n} p_{m}=\rho^{n} \frac{(m \rho)^{n}}{n !} p_{0}
\end{aligned}
$$

Therefore, the probability of zero entity in system $P_{\mathrm{o}}$ based on $\mathrm{M} / \mathrm{M} / \mathrm{m}$ model for single channel queue type as stated by [16] and [25] is given as Eq. (18);

$$
P_{o}=\left[1+\sum_{n=1}^{m-1} \frac{(m \rho)^{n}}{n !}+\frac{(m \rho)^{m}}{m !} \cdot \frac{1}{(1-\rho)}\right]^{-1} \text { for } m \mu
$$

where, $n$ is the number of passengers in system. The probability that an arrival joins queue due to busy servers $P_{q}$, is given as Eq. (19);

$$
P_{q}=\frac{(m \rho)^{m}}{m !} \cdot \frac{P_{o}}{(1-\rho)} \quad n>m
$$

The probability of having $n$ passengers on queue $P_{n}$, is given as Eq. (20) and Eq. (21);

$$
\begin{aligned}
& P_{n}=\frac{(m \rho)^{n} P_{o}}{n !} \quad n \leq m \\
& P_{n}=\frac{(m \rho)^{n} P_{o}}{m^{n-m} m !} \quad n \geq m
\end{aligned}
$$

Such that average waiting time on queue $W_{q}$, is expressed as Eq. 22;

$$
W_{q}=\frac{1}{\mu} \frac{(m \rho)^{m}}{m !} \times \frac{P_{o}}{m(1-\rho)^{2}}
$$

The average waiting time (or average time spent) in system $W_{s}$, is the sum of service time and waiting time on queue. Its expression is given as Eq. (23);

$$
W_{s}=\frac{1}{\mu}+\frac{1}{\mu} \frac{(m \rho)^{m}}{m !} \cdot \frac{P_{o}}{m(1-\rho)^{2}}
$$

Using Little's law which states that, for a steady state queuing system, the number of customers $\mathrm{N}$ on queue in the queuing system at any time is the product of waiting time $\mathrm{W}$ in the system and arrival rate $\lambda$ expressed as Eq. (24);

$$
\mathrm{N}=\lambda W
$$

The average queue length $L_{q}$, is therefore, the product of arrival rate and waiting time on queue in the system which is expressed as Eq. (25);

$$
L_{q}=\lambda W_{q}=\frac{\lambda}{\mu} \frac{(m \rho)^{m}}{m !} \cdot \frac{P_{o}}{m(1-\rho)^{2}}
$$

The number of customers in queuing system is the sum of customers waiting on queue and the serving customers. Therefore, Average number of customers in the system is estimated as Eq. (26);

$$
\mathrm{N}=\lambda W_{s}=\frac{\lambda}{\mu} \frac{(m \rho)^{m}}{m !} \cdot \frac{P_{o}}{m(1-\rho)^{2}}+\frac{\lambda}{\mu}
$$

Any queuing system where $\rho>1$ is described as an unsteady system $[9,23,26-30]$. This implies that, the queue length continues indefinitely [3].

Unsteady queues are best analysed using discrete time or stochastic models to estimate time dependent performance of the system. Monte Carlo simulations and differential equations are required for examining time dependent behaviour of unsteady queuing systems [29].

\section{MATERIALS AND METHODS}

\subsection{Data Description}

This study adopted travel demand data for Manchester and Leeds-Bradford airports from the United Kingdom Civil Aviation Authority database. It measured travel demand in Million Passengers Per Annum (mppa) as 22.0 mppa and 3.3 mppa respectively [7, 8, 32]. Estimated average hourly travel demand measured in persons (pers) per hour for Manchester and LeedsBradford airports were 2511.0 (pers/hour) and 377.0 (pers/hour) respectively.

\subsection{Analytical Model (AM)}

Queuing performance parameters were estimated using formulae established in Section 1.2 above for an $\mathrm{M} / \mathrm{M} / \mathrm{m}$ model to estimate average input parameters for analytical model (AM).

\subsection{Simulation Model (SM) using SimEvents Toolbox}

Simulation technique is an approximation of numerical integral which provides different results for different scenarios and methods. It attempts to ingest the 
difficulties and impact of constraints that favour the mathematical model in numerical analysis rather than reality [33]. This study implements an analytical model using SimEvents a discrete time event tool to examine the performance of queuing system [34].

SimEvents is a MATLAB time or event based toolbox which provides discrete-event simulation engine and component library containing predefined blocks with different system functionalities such as entity generators, FIFO and Priority queue rules, Signal Scope, path combiner, set attribute, etc. used for modelling [14].

A SimEvents model was built using built-in blocks from the Simulink library. Blocks used for this model and their functions are as follows;

i. Event-Based random number - this block is used for setting inter-arrival time for random events to conform with selected arrival distribution type which could be exponential (as specified by this study), uniform, constant etc.

ii. Time-Based entity generator - the block generates discrete entities representing stochastic arrivals based on specified intergeneration time from the Event-Based Random Number block. Intergeneration time is the time lapse between consecutive arrivals in the model. In this model, an exponential distribution type is specified with estimated mean inter-arrival time as the intergeneration time based on demands. The initial seed value of each run is set using 5 digits odd numbers to aid repeatability of random process when re-entered.

iii. FIFO block - this block ensures that entities arriving on the queue willing to proceed to service facility do so based on First-In-First-Out rule. iv. $\quad N$-Server block - this block allows the assignment of more than one server per queue category.

v. Read timer block - it measures arrival and departure time of entities into the system, hence, two sets of it are used to estimate time spent by entities in the system (sojourn time).

vi. Entity Sink - all departures exit the network through entity sink block.

vii. Signal scope - this block displays output of the simulation process in graphical form.

A layout of the built simulation model is as shown in Fig. 1.

\subsection{Estimation of Models Inputs}

Using daily average travel demand in persons per hour as estimated above, a 6 hours' time period of full operation taken as peak period is adopted for this analysis; that is a non-stop inflow and continues service situation lasting for 6 hours at the airports from 05:00 am to 11:00 am. Both AM and SM have different input formats; while AM requires arrival and service rates, inputs for SM are average Inter-arrival time and average service time. Measurement units are such that, the 6 hours' duration of experiment is called 'period' and number of arrivals measured in persons (pers).

\section{RESULTS AND DISCUSSION}

\subsection{Utilisation Factor (UF)}

This is the percentage of time the system actually worked out of available time period for the experiment. It influences other performance parameters of the system [18]. The UF for randomly selected number of scanners for Manchester and Leeds-Bradford airport are as shown in Figs. $2 \mathrm{a}$ and $2 \mathrm{~b}$ respectively.

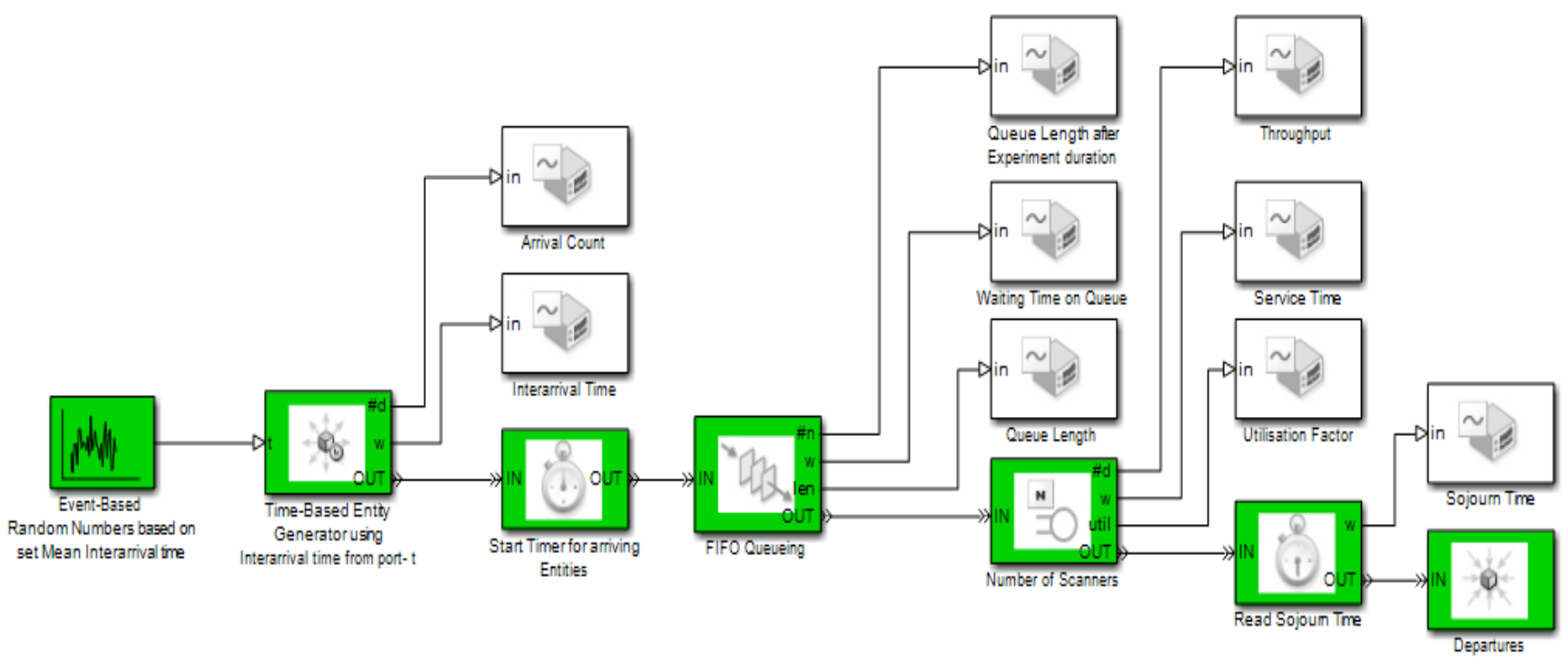

Fig. 1: Simulation Model 


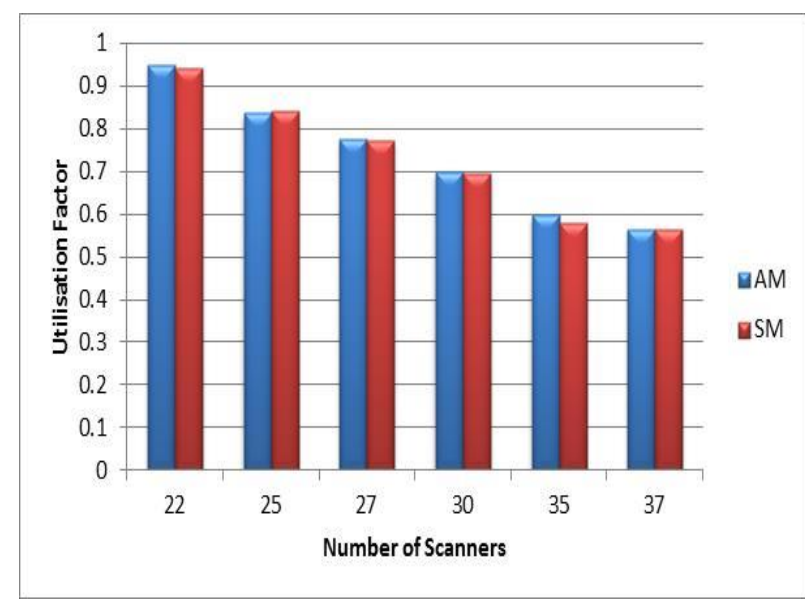

Fig. 2a: UF for Manchester Airport

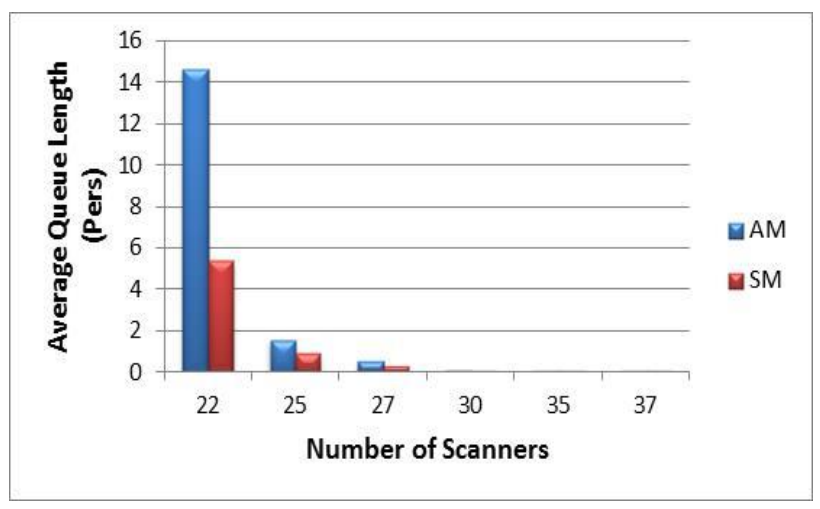

Fig. 3a: Average queue length for Manchester airport

Random selection of scanners is such that minimum number used satisfies the condition $m \mu>\lambda$ to ensure steady state system. A chi-square statistics test at $95 \%$ confidence level showed that there was no significant difference between outputs of AM and SM for both airports with $\chi_{\text {cal. }}^{2}=0.072$ and $\chi_{\text {cri. }}^{2}=11.070$ at 5 degree of freedom for Manchester Airport, and $\chi_{\text {cal. }}^{2}=$ 0.378 and $\chi_{\text {cri. }}^{2}=9.488$ at at 4 degree of freedom for Leeds-Bradford Airport. This indicated that AM and SM models are potentially similar for both airports based on results of UF parameter. There is substantially large difference in working period and number of scanners used. For every increase or decrease in number of scanners used, there is corresponding decrease or increase in UF respectively. This implies that at equal arrival rates, less number of scanners creates high occupancy than high number of scanners in the system [35],[36].

\subsection{Average Queue Length}

This is the number of travellers waiting for service on queue in the system. Figs. $3 \mathrm{a}$ and $3 \mathrm{~b}$ are plots of average queue length generated for different number of

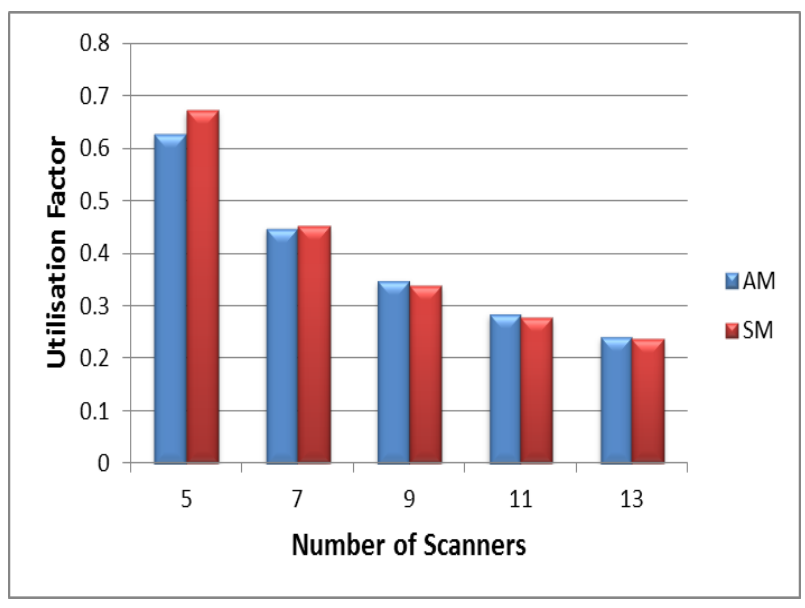

Fig. 2b: UF for Leeds-Bradford Airport

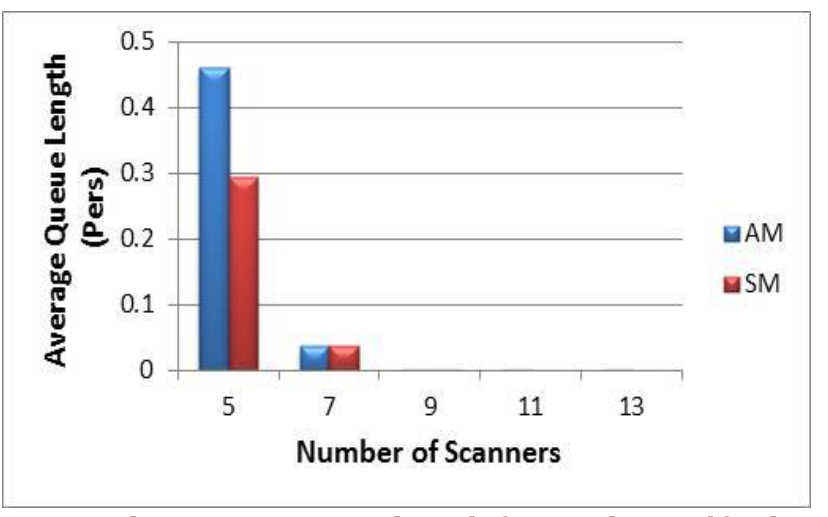

Fig. 3b: Average queue length for Leeds-Bradford Airport

scanners for both AM and SM based on travel demands for Manchester and Leeds-Bradford airports.

Figure 3a revealed that, there was substantial difference of $63.30 \%$ in average queue length between AM and SM using 22 scanners, $56.44 \%$ for 25 scanners and $51.73 \%$ for 27 scanners with AM estimating relatively high values. There was no noticeable queue length for 30, 35 and 37 number of scanners, which was due to sufficient services to handle the demand [9], [20]. Significant variation in average queue length is attributed to assumptions of constant input parameters such as inter-arrival time used by the AM which does not depict reality. This also explained similar variations observed for Leeds-Bradford airports as shown in Fig. 3b. The simulation model considered arrivals at discrete inter-arrival time with unsteady arrivals at the initial stage [21],[34], which leads to time lost or gain in the process before stability was installed as shown in Fig. 4a.

Figure $4 \mathrm{a}$ showed unstable inter-arrival time which lasted till 122th minutes of simulation period for Manchester Airport before stability was installed. Like the Manchester airport, significant variations in parameters for Leeds-Bradford as shown in Fig. 4b were also attributed to relatively high variation in 
inter-arrival times - deviation from mean at the beginning of the process. Random arrivals never assume average inter-arrival time as shown in Fig. $4 \mathrm{~b}$ in the experiment. This was attributed to relatively less demand from Leeds-Bradford airport which is spatially shared within experimental duration.

Also, considerable difference in queue length and long queue lengths could be attributed to estimation errors due to system instability caused by high utilisation factor [29], and insufficient services to cater for demand respectively [35],[36].

\subsection{Average waiting time on queue}

This is time spent on queue by travellers waiting for services. It could be established that average waiting

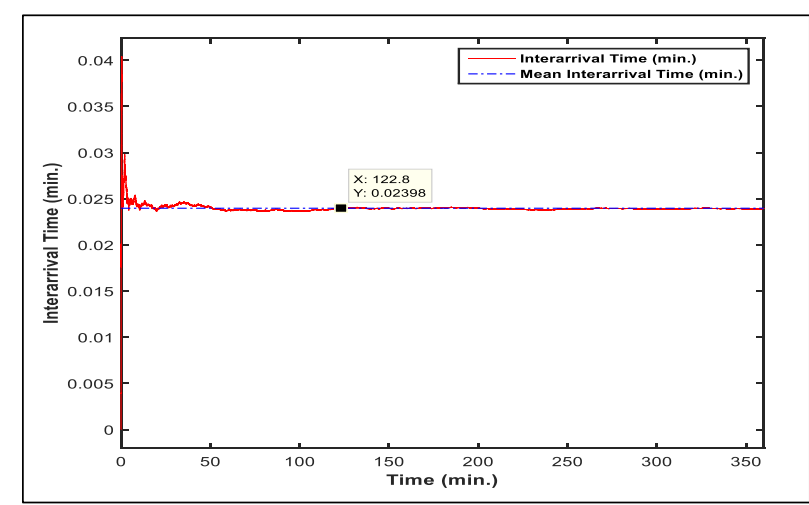

Fig. 4a: Simulated inter-arrival time for Manchester airport

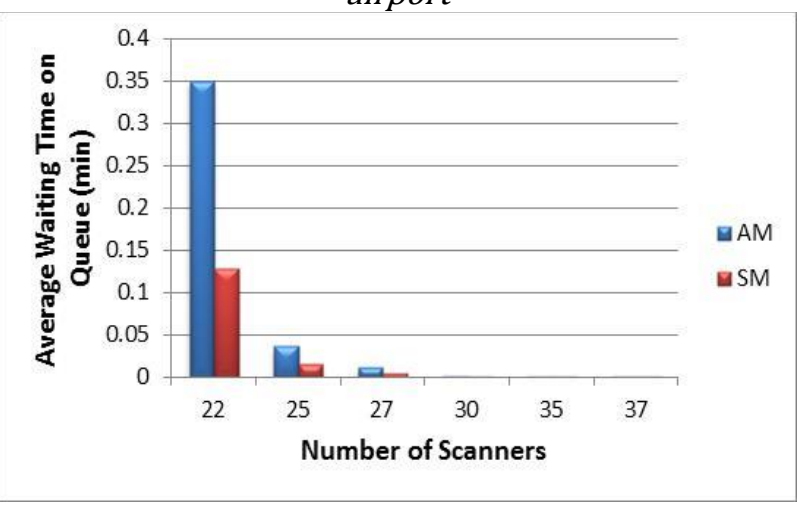

Fig. 5a: Average waiting time on queue for Manchester airport time on queue was directly proportional to queue length. This is best explained using Fig. 5a which presents similar trend with average queue length for Manchester airport.

Figure 5a revealed significant difference of $63.24 \%$ between $\mathrm{AM}$ and $\mathrm{SM}$ for 22 scanners, $56.39 \%$ difference for 25 and $51.67 \%$ for 27 scanners. These differences maintained a trend as above (Section 3.2). There is no noticeable waiting time on queues for 30 , 35 and 37 number of scanners; this is attributed to sufficient supply of services. Characteristics of this plot are same as those of Section 3.2., hence assumes similar explanation of constant and varied input parameters for AM and SM respectively for Manchester Airport.

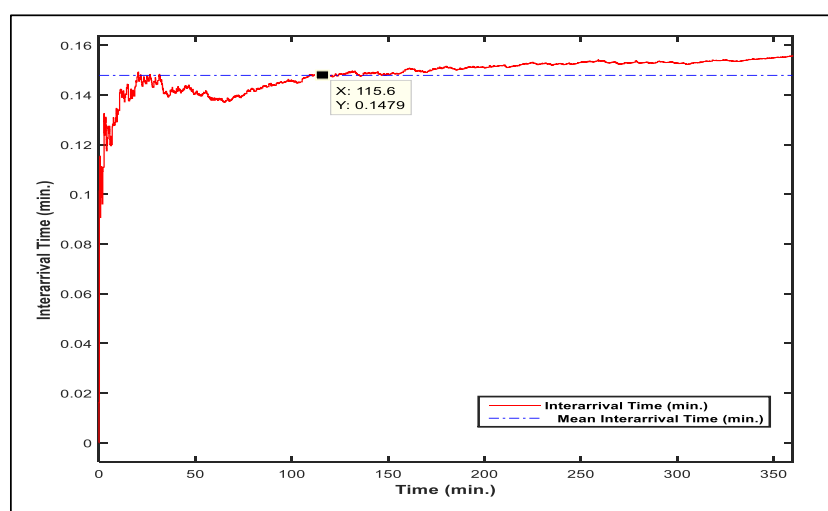

Fig. 4b: Simulated inter-arrival time for Leeds-Bradford Airport

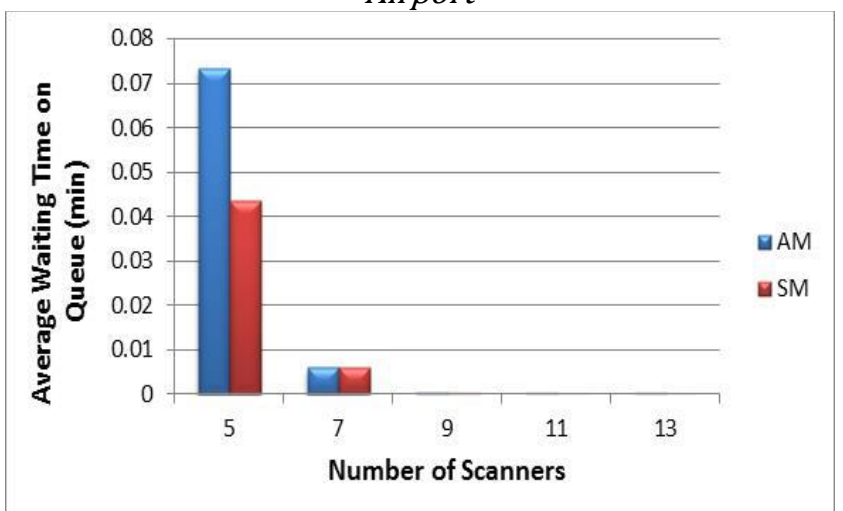

Fig. 5b: Average waiting time on queue for LeedsBradford Airport

Table 1: Estimations of passenger flow using AM and SM for Manchester Airport

\begin{tabular}{llllllc}
\hline \multirow{2}{*}{ Number of Scanners } & \multicolumn{3}{c}{ Models } \\
\cline { 2 - 7 } & \multicolumn{3}{c}{ Analytical (AM) } & \multicolumn{3}{c}{ Simulated (SM) } \\
\cline { 2 - 7 } & A (pers) & T (pers) & N (pers) & A (pers) & T (pers) & N (pers) \\
\hline 25 & 15069 & 15033 & 36 & 15030 & 15010 & 6.3470 \\
27 & 15069 & 15047 & 22 & 15350 & 15330 & 1.3400 \\
30 & 15069 & 15048 & 21 & 15100 & 15080 & 0.8704 \\
35 & 15069 & 15048 & 21 & 15110 & 15090 & 0.5481 \\
37 & 15069 & 15048 & 21 & 14920 & 14890 & 0.5070 \\
\hline
\end{tabular}


On the other hand, Leeds-Bradford airport experienced average waiting time on queue in the system as shown in Fig. 5b, which assumes similar features as explained for Manchester airport. Since these plots maintain similar trend, time spent in system (sojourn time) is the sum of waiting time and service time.

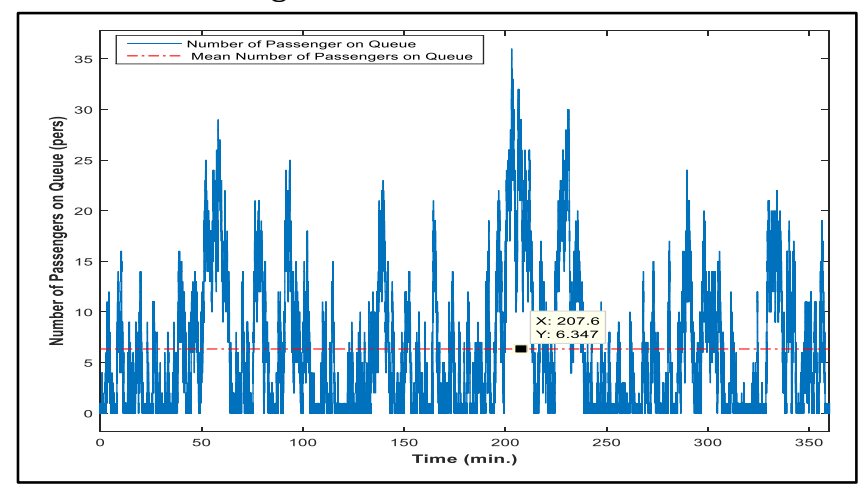

Fig. 6: Estimation of queue length at the end of experiment

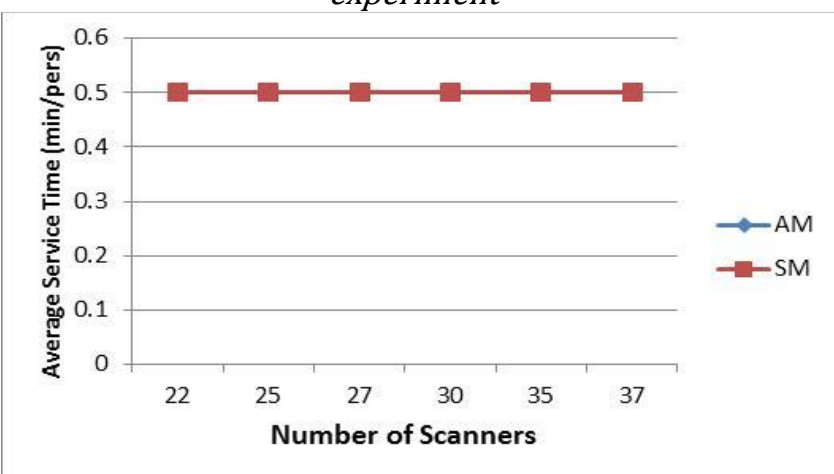

Fig. 7: Average service time for Manchester airport

\subsection{Average Arrivals and Throughputs}

These are the inflow and outflow of travellers in the system respectively. Analysis of arrival inflow and outflow for Manchester airport is as shown in Table 1; while AM adopted constant inflow based on estimations from demand data, the SM being a random process is set to have finite capacity to accommodate only estimated demand within experimental period. Results for maximum arrival (A), maximum throughput (T) and average number of passengers on queue after experimental period (N) for AM and SM for the Manchester airport are as shown in Table 1.

Average queue length after the $6^{\text {th }}$ hour (360 minutes) is the difference between total arrivals and throughput [3]; both AM and SM estimates average parameters. Results obtained from SM were read from discrete time plot of number of passengers on queue after simulation period. The results revealed that there was significant variation between output of $\mathrm{AM}$ and $\mathrm{SM}$, this is attributed to assumptions of a deterministic system for the AM and stochastic analysis used in SM. SM outputs are estimated from balancing high range of variations during the process as shown in Fig. 6 which leads to significant difference from AM output.

Also, the maximum number of passengers on queue at the end of simulation process could be wrongly estimated by the SM; since maximum value may not actually occur at the end of the experiment but within experimental period (about 208 $8^{\text {th }}$ minutes) as shown in Fig. 6.

Though SM did not necessarily balance the arrival minus departure arithmetic, the process considered arrivals less or greater than specified - finite number. This is attributed to variations in randomised and probabilistic processes which might ignore excess arrivals or overestimate arrivals at the end of simulation period specified, unlike AM which assumes constant input parameters that also leads to under or over estimations [23, 30]

It has been established that both models used equal UF; a plot of average service time for both models as shown in Fig. 7 indicates equality in average service time per arrival for both models.

Since execution of AM adopts constant average service time, the major input parameter that could create difference in results of the process was potential variations in inter-arrival time which could be easily examined by discrete time process using SM [37]. On the other hand, arrival inflow and outflow for Leeds-Bradford airport were as shown in Table 2.

Table 2: Passenger flow estimations using AM and SM for Leeds-Bradford Airport

\begin{tabular}{ccccccc}
\hline & \multicolumn{5}{c}{ Models } \\
\cline { 2 - 7 } Number of Scanners & \multicolumn{3}{c}{ Analytical (AM) } & \multicolumn{3}{c}{ Simulated (SM) } \\
\cline { 2 - 7 } & A (pers) & T (pers) & N (pers) & A (pers) & T (pers) & N (pers) \\
\hline 5 & 2262 & 2258.4 & 3.6027 & 2310 & 2309 & 0.7379 \\
7 & 2262 & 2258.8 & 3.1797 & 2272 & 2270 & 0.5373 \\
9 & 2262 & 2258.9 & 3.1446 & 2211 & 2207 & 0.5031 \\
11 & 2262 & 2258.9 & 3.1418 & 2263 & 2260 & 0.4999 \\
13 & 2262 & 2258.9 & 3.1417 & 2265 & 2261 & 0.4999 \\
\hline
\end{tabular}


From Table 2, both AM and SM yielded results with substantial differences. AM estimates relatively high number of passengers left on queue after experimental period. This conforms with facts earlier established for Manchester airport. The slight difference of $0.04 \%$ between service time for Leeds-Bradford airport as shown in Fig. 8 were related to differences in service time which was not significant and potentially caused by low demand hence assumed to have no impact on these models.

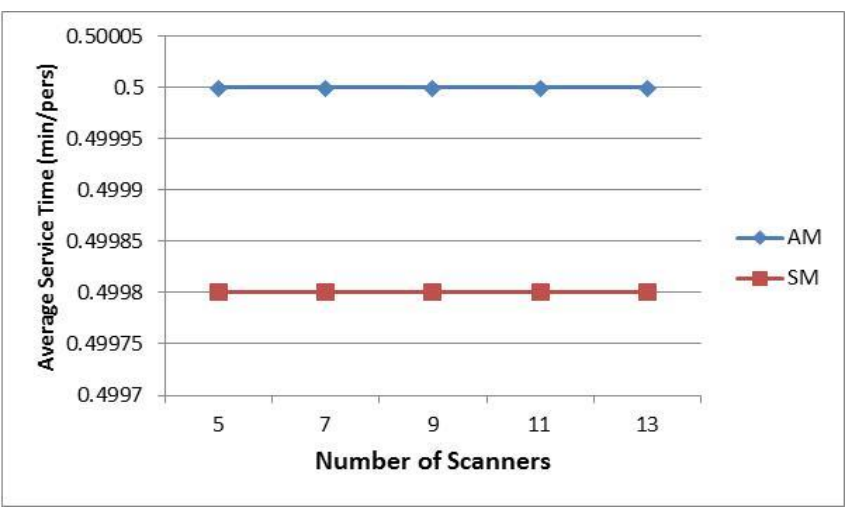

Fig. 8: Average service time for Leeds-Bradford airport

\section{CONCLUSION}

This study implemented AM developed by previous researches using simulation toolbox called SimEvents in MATLAB, based on travel demand data for Manchester and Leeds-Bradford airports in the United Kingdom. Results obtained from AM and SM for both airports indicated that both models actually worked for approximately equal durations using equal service time per arrival as confirmed by chi square statistic test on UF parameters and average service time plot respectively. It was established that the AM and SM models actually considered equal service period for scanners with approximately equal service time which conventionally showed similarity in operations; the similarity in UF and service time between the models clearly showed how time was shared for activities in the system. Therefore, it was presumed that both models are potentially equal with negligible intermediate variations. Though other output parameters of the models such as average queue length, average waiting time on queue and waiting time in the system and maximum throughput did not indicate good fit of the models. This was attributed to variations in discrete time events considered by SM in which the AM assumed constant values during experimental period, which affected the accuracy of estimations. The SM exhibited stochastic behaviour which actually depicts reality hence yielded reliable results. It was therefore concluded that, simulation results are preferred and recommended for airport managers since they are more realistic based on discrete time analysis.

\subsection{RECOMMENDATIONS}

Significant variations in model outputs for queue performance due to assumptions of constant input parameters for AM and discrete time analysis of SM could be improved by using discrete time analysis techniques such as; differential equations used in Markov chain method for discrete time analysis for better outputs. The SM is capable of giving airport managers a prior knowledge of the system's behaviour hence recommended for proper planning and service improvement in terms of service configuration and discipline for a better LOS at airports.

\section{ACKNOWLEDGMENTS}

I am grateful to the UK Civil Aviation Authority for giving me access to their air travel demand data. Also, I wish to appreciate the efforts of Dr. Richard Connors of the Institute for Transport Studies (ITS), University of Leeds UK whose assistance made this work successful.

\section{REFERENCES}

[1] Ahyudanari, E. and Vandebona, U. "Simplified Model for Estimation of Airport Check-In Facilities", Journal of the Eastern Asia Society for Transportation Studies, Vol. 21, No. 9, pp. 724 735, 2005.

[2] Hillier, F. S. and Lieberman, G. J. Introduction to Operations Research, $7^{\text {th }}$ Edition, McGraw Hill. Companies, Inc., New York, 2001.

[3] Jain, J. L, Mohanty, S. G. and Bohm, W. A Course on Queueing Models, Statistics: A series of Textbooks and Monographs, Chapman \& Hall/CRC, Taylor \& Francis Group, 2007.

[4] Bastani, P. "A Queueing Model of Hospital Congestion", MSc. Thesis submitted to Department of Mathematics Simon Fraser University, 2009.

[5] Copper, R. B. Introduction to Queueing Theory, $2^{\text {nd }}$ Edition North Holland, 1981.

[6] Gross, D. and Harris, C. M. Fundamentals of Queueing Theory, 2nd ed. John Wiley \& Sons: New York, 1985.

[7] Department for Transport "UK Aviation Forecast", London. 2013, www.gov.uk/dft, Accessed on June 14, 2015. 
[8] Eurostat "Air Transport of Passengers", http://ec.europa.eu/eurostat/data/database, Accessed on June 17, 2015.

[9] Train, K. Discrete Choice Methods with Simulation, Cambridge University Press. UK Civil Aviation Authority, 2015. Passengers at UK airports increase for the fourth year in a row, 2002, www.caa.co.uk, Accessed on April 17, 2015.

[10] Czerny, A. “Airport Congestion Management under Uncertainty", Transportation Research Part B, Vol. 44, No. 3, pp. 371 - 380, 2010.

[11] Manataki, I. E. and Zografos, K. G. "A Generic System Dynamics based Tool for Airport Terminal Performance Analysis. Transport Research Part C, Vol. 17, No. 4. Pp. 428 - 443, 2009.

[12] Abdelghany, A. and Addelghany, K. "Modelling Applications in the Airline Industry", ASHGATE, England. 2012.

[13] Fitzsimmons, J. A. and Fitzsimmons, M. J. Service Management - Operations, Strategy, Information Technology, McGrowHill, 2004.

[14] MATLAB “User Guide Manual”, 2016.

[15] Brunetta, L., Righi, L. and Andreatta, G. "An Operations Research Model for the Evaluation of an Airport Terminal: SLAM (Simple Landside Aggregate Model), Journal of Air Transport Management, Vol. 5, No. 3, pp. 161 - 175, 1999.

[16] Adan, I. and Resing, J. "Queueing Systems", Department of Mathematics and Computer Science, Eindhoven University of Technology, Netherlands, 2015.

[17] Kleinroc, L. and Finelstein, R. P. "Time Dependent Priority Queues", Operations Research, Institute for the Operations Research and Management Science, Vol. 15, No. 1, Pp. 104-116, 1967.

[18] Sztrik, J. Basic Queueing Theory, University of Debrecen, Faculty of Informatics, 2012.

[19] Bunday, B. D. An Introduction to Queueing Theory. Arnold: London, 1996.

[20] Coffman, E. G. and Denning, P. J. "Operating Systems Theory", Prentice-Hall, Inc., Englewood Cliffs, N.J. USA, 1973.

[21] Stephen, F. F. "Two Queues under Preemptive Priority with Poisson Arrival and Service Rates", Operations Research, Vol. 6, No. 3, pp. 399-418, 1958.

[22] Bunday, B. D. "Mathematical Modelling of Queues, the Mathematical Association", The Mathematical Gazette, Vol. 79, No. 486, pp. 499-512, 1995.

[23] Warthington, D. and Wall, A. "Using the Discrete Time Modelling Approach to Evaluate the Timedependent behaviour of Queueing Systems", The Journal of the Operational Research Society, Vol. 50, No. 8, pp. 777-788, 1999.
[24] Ibe, 0. C. Fundamental of Applied Probability and Random Processes, Elsevier Academic Press, USA, 2005.

[25] Saidi, S. and de Barros, A. "Assessing Airport Passenger Screening Processing Systems", $13^{\text {th }}$ World Conference on Transport Research (WCTR), July 15 - 18. 2013.

[26] Bhat, U. N. "Sixty Years of Queueing Theory", Management Science, Vol. 15, No. 6, 1969.

[27] Bordenave, C. "Stability of Spatial Queueing Systems", Advances in Applied Probability, Vol. 38, No. 2. pp. 487-504, 2006.

[28] Gail, H. R., Hantler, S. L. and Taylor, B. A., "Analysis of a Non-preemptive Priority Multi Server Queue", Advances in Applied Probability, Applied Probability Trust, Vol. 20, No. 4, pp. 852-879, 1988.

[29] Osogami, T., Wierman, A., Harchol-Balter, M. and Scheller-Wolf, A. "How many servers are best in a Dual-priority FCFS System?" Technical Report, School of Computer Science, Carnegie Mellon University, 2003.

[30] Simmons, R. L. "A Queueing Theory Application with Time-dependent Parameters, Agricultural \& Applied Economics Association", Journal of Farm Economics, Vol. 43, No. 5, 1961, pp. 1408-1409, www.jstor.org/stable/1235616, Accessed on March 2, 2015.

[31] Zhao, Y. and Grassmann, W. "Queueing Analysis of a Jockeying Model”, Operations Research, Vol. 43, No. 3, 1995, pp. 520 - 529.

[32] Adeke, P. T. "Mathematical Modelling of Priority Queues", Unpublished M.Sc. Dissertation, University of Leeds. UK. 2015.

[33] Trakoonsanti, L. "A Process Simulation Model of Airline Passenger Check-In", Universal Journal of Management, Vol. 4, No. 5, pp. 265-276, 2016.

[34] Worthington, D. "Reflections on Queue Modelling from the Last 50 Years", The Journal of the Operational Research Society, 2009, pp. 583-592, www.jstor.org/stable/40206727, Accessed on February 23, 2015.

[35] Bruno, G. and Genovese, A. "A Mathematical Model for the Optimisation of the Airport Check-in Service Problem", Electronic Notes in Discrete Mathematics, Vol. 36, pp. 703-710, 2010.

[36] Ha, A. Y. "Incentive-Compatible Pricing for a Service Facility with Joint Production and Congestion Externalities", Management Science, Vol. 44, No. 12, Part 1 of 2, pp. 1623-1636, 1998.

[37] Ross, S. Stochastic Processes, John Wiley and Sons, New York, 1983. 